\title{
Universidad de Guadalajara y regionalidad: un proyecto educativo para el occidente y noroeste de México
}

DOI: https://doi.org/10.32870/dse.v0i11.276

\section{María Guadalupe García Alcaraz* Luciano Oropeza Sandoval $^{\star *}$}

Resumen. En este escrito se describe el espacio regional que construye la Universidad de Guadalajara con varias entidades federativas a partir de la relación entre su propuesta educativa y la procedencia geográfica de los alumnos. Este fenómeno es entendido como el vínculo que se establece entre la oferta educativa que ofrece esta universidad y el lugar de origen de los estudiantes. El estudio abarca desde 1925 hasta 1981, lapso en el que se amplía la oferta educativa y se establecen universidades estatales en la mayoría de las entidades del país. Estos hechos nos llevan a postular que dicha regionalidad se ve delineada por la diversificación que aparece en la oferta educativa, por los cambios en los intereses educativos de los estudiantes y por la gradual creación de universidades públicas. Palabras claves: Universidad de Guadalajara, egresados, procedencia geográfica y espacio regional.

\begin{abstract}
In this paper we describe the regional space that form the Universidad de Guadalajara with some states as from the relation among it educative proposal and the geography origin of the students. This event is understood as the link that it found among the educative offer that propose this university and the origin space of the students. This paper cover from 1925 to 1981 , lapse in that itself extend the educative offer and establish states university in the majority of the states from Mexico. About the matter, we pose that aforementioned regionalization itself delineates out of la diversification that emerge in the offer educative, the changes in educative expectations of the students and by the creation gradual of the public universities. Keywords: Universidad de Guadalajara, graduates, geography origin and regional space.
\end{abstract}

\section{La construcción de la región}

Para fundamentar el espacio regional que se configura entre la Universidad de Guadalajara y la población escolar que ingresa y concluye sus estudios profesionales, se acude a las ideas vertidas en torno al concepto de región. Los estudiosos del tema señalan como premisa inicial que este concepto no es una categoría transhistórica, ya que su significado se erige a partir de circunstancias de tiempo y lugar y de las particularidades de la problemática que se desea abordar.

El concepto de región es una construcción social que los académicos elaboran a partir de los requerimientos teóricos y metodológicos que plantea el análisis de una problemática específica (Padilla Arroyo, 2006). Esta construcción puede identificarse con una definición funcional de un espacio geográfico con fronteras bien delimitadas, con divisiones políticas o administrativas pre-

* Doctora en Ciencias de la Educación. Profesora-investigadora titular "CC" del Departamento de Estudios en Educación de la Universidad de Guadalajara. Correo electrónico: gaag4@cencar.udg.mx

***Doctor en Ciencias de la Educación. Profesor-investigador titular "C" del Departamento de Estudios en Educación de la Universidad de Guadalajara. Correo electrónico: loropezasandoval@yahoo.com.mx 
existentes, o con los rasgos topográficos de una zona específica; pero también puede definirse como un espacio geográfico con fronteras determinadas por el alcance efectivo de alguna clase de sistema cuyas partes se interrelacionan entre sí más que con otras partes ubicadas fuera del sistema (Van Young, 1989).

Apoyados en estas ideas, en especial en aquellas que señalan la posibilidad de construir un concepto de región a partir de la interrelación de varios elementos de un sistema social, delimitamos nuestra concepción desde la relación que se establece entre el proyecto educativo que ofrece la Universidad de Guadalajara y el lugar de origen de los estudiantes que concluyen estudios de nivel superior (Castañeda, 1994; Martínez Moctezuma, 2010). Esta relación, no sobra decirlo, no incluye los lugares donde estos individuos desarrollan su actividad profesional.

Este fenómeno es examinado en el periodo comprendido entre 1925 y 1981. Se parte de 1925 porque es el año de la refundación de la Universidad de Guadalajara, y termina en 1981 porque los acervos documentales que resguardan los archivos históricos sólo abarcan hasta treinta años previos al año vigente.

\section{Origen de los alumnos}

Para conocer el lugar de procedencia de los hombres y mujeres que ingresaron a la Universidad de Guadalajara desde 1925 hasta 1981 se consultaron los fondos documentales del Archivo Histórico de la Universidad de Guadalajara (AHUG). Ahí se revisó el acervo denominado Series Profesionales, donde se concentran expedientes relativos a la tramitación del título profesional. Estos expedientes proporcionaron la materia prima para elaborar gráficas relativas al lugar de origen de los alumnos y alumnas que ingresaron a la universidad en el periodo señalado. Esta información no representa la totalidad de la población escolar sino sólo aquella proporción que tramita su título profesional. Sin embargo, creemos que la muestra permite detectar las tendencias que se expresan en torno a la procedencia geográfica.

La organización de esta información se realiza a partir de dos criterios: de la existencia de uniformidad en los datos y de la representatividad en torno a la población total. En esa tónica, la información inicial abarca todo el primer lustro de la universidad debido a que hay cierta irregularidad en los datos de titulación que se presentan año tras año.

A partir de 1938 aparece más regularidad en la frecuencia de casos de titulación por año, hecho que permite seleccionar una proporción temporal por cada década. Así, se procedió a tomar los dos primeros años de los decenios que van de 1940 a 1980. Esta muestra representa entre 15 y $20 \%$ del total de los egresados en cada una de estas décadas.

La información que exponemos en este periodo se organiza conforme a la procedencia geográfica. Así, se elaboraron las agrupaciones siguientes: a) Guadalajara, b) interior de Jalisco, c) estados vecinos, donde se incluyen Colima, Michoacán, Guanajuato, Zacatecas y Aguascalientes, d) 
estados del noroeste, que abarca a Nayarit, Sinaloa, Sonora, Baja California y Baja California Sur. En esta última agrupación se integra a Nayarit, a pesar de ser una entidad vecina a Jalisco, debido principalmente a su ubicación en el noroeste del país.

\section{Los primeros años: una espacialidad limitada a la entidad y los estados vecinos}

El 12 de octubre 1925 es reinaugurada la Universidad de Guadalajara, iniciativa que permite darle estructura orgánica a diversas escuelas y facultades que existían desde el siglo XIX y las primeras décadas del siglo xx. En esa etapa inaugural, su oferta educativa se integra por los siguientes segmentos:

a) Adiestramientos de nivel técnico-artesanal, impartidos en la Escuela Politécnica, creados básicamente para capacitar a las capas trabajadoras; de ahí los pocos requisitos solicitados: un documento que acreditaba la terminación del segundo año de instrucción primaria especial.

b) Carreras ligadas al comercio y los servicios como Tenedor de libros y Comercio (Facultad de Comercio), además de Enfermería y Obstetricia, anexas a la Facultad de Medicina; el requisito principal para ingresar a cada una de ellas es el certificado de instrucción primaria superior (con la excepción de Obstetricia, donde se requiere previamente los estudios de enfermería).

c) Enseñanza de nivel medio ofrecida en la Preparatoria de Jalisco y en la Preparatoria para Señoritas y Normal Mixta.

d) Carreras de nivel superior impartidas en las facultades de Farmacia, Ingeniería, Jurisprudencia y Medicina; en esta última se localiza inicialmente la carrera de Odontología. ${ }^{1}$

En estos establecimientos se ubican 2764 alumnos en 1925, suma que llegará a cerca de 3000 en 1930. En este lustro, como puede verse en la gráfica siguiente, $74 \%$ de los estudiantes proviene de Guadalajara y de localidades del interior de Jalisco, 12\% de los estados vecinos, $6 \%$ de los estados del noroeste y $10 \%$ de otras entidades federativas y del extranjero.

En esos años, la mayoría de la población estudiantil que proviene de los estados vecinos se concentra principalmente en la carrera de medicina y en proporción menor en las de derecho, farmacia y odontología, como puede verse en la gráfica siguiente:

Esta información permite ver que la espacialidad inicial entre esta institución y los lugares de procedencia se constriñe básicamente al estado de Jalisco y los estados limítrofes. Asimismo,

\footnotetext{
${ }^{1}$ La carrera de Odontología se separa de la Facultad de Medicina en 1927, dando lugar a la Escuela Dental (Oropeza, 1990).
} 
muestra que las expectativas de los alumnos procedentes de esas entidades se concentran básicamente en carreras como medicina, derecho, odontología y farmacia. Este tipo de vínculo con los espacios de origen geográfico se mantendrá a lo largo de los años treinta, aunque sobrevendrán algunos cambios relativos, como se verá enseguida.

\section{La reorganización de la universidad pública}

En los años treinta, la Universidad de Guadalajara se ve afectada por las reformas promovidas al artículo $3^{\circ}$ constitucional; la participación de la comunidad universitaria en torno a las dos principales posiciones que emergen de la orientación ideológica de la educación genera diversas confrontaciones que llevan al gobierno de la entidad a cerrar temporalmente esa institución y a sustituirla por la Dirección General de Estudios Superiores (DGES), organismo que tiene como cometido la administración de los planteles y la eliminación de los disturbios que obstruyen la marcha de la vida universitaria.

En marzo de 1935, la DGES abre las principales escuelas de nivel superior y da continuidad a la labor educativa. Sin embargo, la inestabilidad social que se vive en la entidad debido a las implicaciones derivadas de la reforma del artículo tercero constitucional, afecta la matrícula escolar: de 3 451 alumnos inscritos en el año escolar 1933-1934, se pasa a 1200 en marzo de 1935. Este elevado descenso de la matrícula sólo se recuperará hasta mediados de los años cincuenta. ${ }^{2}$

Casi tres años después, en julio de 1937, se reabre la Universidad de Guadalajara. Esta institución sustituye a la DGES y vuelve a ofrecer los siguientes servicios educativos:

Carreras de enseñanza técnica y comercial impartidas en la Escuela Politécnica y en la Escuela de Comercio y Administración, respectivamente. En esta agrupación también se ubican las carreras de enfermería y obstetricia, aún dependientes de la Facultad de Medicina.

a. Enseñanza media y media superior proporcionada por la secundaria para varones, la secundaria para mujeres y la preparatoria de Jalisco.

b. Enseñanza de nivel superior impartida en las facultades de Ciencias Físicomatemáticas, Ciencias Médicas y Biológicas, Ciencias Químicas, Derecho y Economía, y Odontología.

Entre esta reapertura y los años sesenta, se emprenden acciones educativas que contribuyen a delinear algunos rasgos fundamentales de la Universidad de Guadalajara. Por ejemplo, se reestructuran las carreras vinculadas a la farmacia y se crean otras opciones en la Facultad de Ingeniería, se excluye la enseñanza secundaria, se delimita la educación técnica, se amplía la cobertura educativa de nivel medio superior, se uniforman los requisitos de ingreso a las carreras de nivel superior y se inauguran

\footnotetext{
${ }^{2}$ En el año escolar 1955-1956, la matrícula escolar ascendería a 3739 alumnos (Oropeza, 1990)
} 
nuevos centros educativos. Estos cambios contribuyen a darle fisionomía a la Universidad de Guadalajara, porque se delimitan los niveles educativos correspondientes a su jurisdicción, se precisa la gradación de las carreras de nivel medio superior y superior y se crean carreras que puntualizan el perfil de la educación pública.

En ese lapso aparecen ligeras variantes en la procedencia geográfica de los alumnos. En la gráfica siguiente se puede ver que, al inicio de los años cuarenta, hay un decrecimiento relativo de la población escolar que provenía de Guadalajara y del interior de Jalisco, al pasar de 74\% a 64\%. En contraste, la participación relativa de los estudiantes de los estados del noroeste se eleva de 5.9\% a $10 \%$. A su vez, la proporción de egresados de los estados vecinos prácticamente se mantiene — de $12 \%$ pasa a $13 \%$ -

Al comienzo de esa década, como se observa en la gráfica siguiente, $83 \%$ de los alumnos procedentes de los estados vecinos y del noroeste del país se concentra principalmente en tres carreras: en las de medicina, derecho y enfermería y obstetricia.

En esos años destaca el crecimiento absoluto de la población escolar que proviene de los estados del noroeste del país, como Nayarit, Sinaloa, Sonora y Baja California Sur. Este flujo de migrantes se concentra básicamente en carreras como medicina, derecho y enfermería y obstetricia.

Esta configuración de la espacialidad regional acusa pocos cambios a lo largo de las dos décadas posteriores. En la gráfica siguiente se puede ver que la población proveniente del estado de Jalisco mantiene casi la misma proporción — de 64\% en 1940 pasa a 68\% en 1960—, situación que varía en las otras agrupaciones, ya que el porcentaje de alumnos procedentes de los estados del noroeste sube de $10 \%$ a $13 \%$ y, en contraste, la proporción de los estados vecinos baja de $13 \%$ a $8 \%$.

En relación con las preferencias educativas encontramos cierta continuidad, pero también visibles diferencias entre los jóvenes que provienen de los estados vecinos y de los estados del noroeste. En 1960, 44\% de los estudiantes procedentes de los estados vecinos ingresa a la carrera de medicina, $16 \%$ a derecho, $15 \%$ a ingeniería mecánica electricista y $25 \%$ se distribuye en cuatro carreras más.

Los alumnos procedentes de los estados del noroeste también concentran su interés en la carrera de medicina, aunque en menor proporción. En la gráfica siguiente se puede ver que $27 \%$ ingresa a esta carrera, $16 \%$ a odontología y $16 \%$ a ciencias químicas. Casi tres de cada cinco ingresan a estas tres carreras y cerca de $40 \%$ se distribuye en cinco carreras más.

La información anterior muestra que la espacialidad regional de la Universidad de Guadalajara se extiende hacia los estados del noroeste del país. Su propuesta educativa ya no sólo abarca los linderos del estado de Jalisco y las entidades limítrofes, sino también los estados de Nayarit, Sinaloa, Sonora y Baja California Sur. Cabe destacar que en esta expansión de su cobertura regional la carrera de medicina sigue siendo el atractivo fundamental, aunque se observa una creciente diversificación de las opciones que eligen los estudiantes. 


\section{La creación de universidades estatales y la expansión de la matrícula educativa}

Entre los años sesenta y setenta del siglo xx sobrevienen algunos sucesos que amenazan la estructura de esa espacialidad regional. Por un lado, destaca la creación de universidades públicas en los diversos estados del país y, por otro, la expansión de la oferta de educación superior. Ambos fenómenos inciden en las pautas migratorias de los estudiantes que tradicionalmente provienen de diversos espacios regionales. La apertura de nuevas universidades estatales emerge como un foco atrayente para la población nativa, hecho que entraña una posible disminución de la población que optaba por estudiar en universidades ubicadas en otros estados. A su vez, la diversificación del abanico de carreras ofertadas por la Universidad de Guadalajara también incide en el comportamiento de los flujos migratorios, ya que las nuevas opciones educativas ejercen mayor atracción social. ¿Qué sucede realmente en estas dos décadas? ¿Acaso se modifica la espacialidad regional de la Universidad de Guadalajara? ¿Acaso continúa el flujo migratorio? O, ¿vemos la continuidad de ese flujo migratorio con cambios en las expectativas educativas de los estudiantes? Veamos lo que sucede en esta etapa.

\section{La creación de universidades estatales}

La escolarización de la educación superior ${ }^{3}$ en México es un proceso que prácticamente abarca el siglo xx. Así, pese a que algunas entidades ya contaban con instituciones que brindaban estudios profesionales, la figura secular de universidad, distinta de la vieja universidad colonial, surge a partir de la inauguración de la Universidad Nacional en 1910. Al poco tiempo comienza la apertura de universidades estatales en el país. En el cuadro siguiente podemos ver que la pasarela inaugural inicia con la Universidad Michoacana, organismo creado en 1917. Después aparecen otras más, como la Universidad de Guadalajara, la Universidad de Sonora, la Universidad de Sinaloa y la Universidad de Guanajuato, entre otras.

\footnotetext{
${ }^{3}$ Por escolarización de la educación superior entendemos al proceso histórico a través del cual se organiza la instrucción de los niveles educativos que pasan a formar parte del subsistema universitario. Este proceso incluye la delimitación de la escolaridad de esos niveles, la gradación de las carreras, la delimitación de los conocimientos por niveles y disciplinas, la formación de profesores, los modos de otorgar calificaciones y las formas de graduación, entre otros aspectos.
} 
Cuadro 1: Apertura de universidades estatales 1917-1950

\begin{tabular}{|l|l|}
\hline Nombre de la Universidad & Año de creación \\
\hline Universidad Michoacana & 1917 \\
\hline Universidad del Sureste & 1922 \\
\hline Universidad de San Luis Potosí & 1923 \\
\hline Universidad de Guadalajara & 1925 \\
\hline Universidad de Nuevo León & 1933 \\
\hline Universidad de Puebla & 1937 \\
\hline Universidad de Sonora & 1942 \\
\hline Universidad de Sinaloa & 1942 \\
\hline Universidad Veracruzana & 1944 \\
\hline Universidad de Guanajuato & 1945 \\
\hline Universidad de Coahuila & 1945 \\
\hline Universidad de Querétaro & 1950 \\
\hline
\end{tabular}

Fuente: David Piñera Ramírez (2008). Las universidades de los estados de la república en el contexto nacional.

Para la segunda mitad del siglo xx, como se puede observar en el cuadro siguiente, se crean universidades en el resto de los estados. Así, empezando con la Universidad de Morelos hasta terminar con la apertura de la Universidad de Quintana Roo, todas las entidades federativas llegan a tener su propia universidad. 
Cuadro 2: Apertura de universidades estatales 1951-1991

\begin{tabular}{|l|l|}
\hline Nombre de la Universidad & Año de creación \\
\hline Universidad de Morelos & 1953 \\
\hline Universidad de Chihuahua & 1954 \\
\hline Universidad Juárez de Oaxaca & 1955 \\
\hline Universidad de Campeche & 1955 \\
\hline Universidad de Tamaulipas & 1956 \\
\hline Universidad del Estado de México & 1956 \\
\hline Universidad Autónoma de Baja California & 1957 \\
\hline Universidad de Tabasco & 1958 \\
\hline Universidad Autónoma de Guerrero & 1960 \\
\hline Universidad de Colima & 1960 \\
\hline Universidad Autónoma de Hidalgo & 1961 \\
\hline Universidad Autónoma de Tlaxcala & 1962 \\
\hline Universidad del Carmen & 1965 \\
\hline Universidad Autónoma de Zacatecas & 1968 \\
\hline Universidad Autónoma de Nayarit & 1969 \\
\hline Universidad Autónoma de Ciudad Juárez & 1973 \\
\hline Universidad Autónoma de Aguascalientes & 1974 \\
\hline Universidad de Chiapas & 1975 \\
\hline Universidad de Baja California Sur & 1976 \\
\hline Universidad de Quintana Roo & 1991 \\
\hline
\end{tabular}

Fuente: David Piñera Ramírez (2008). Las universidades de los estados de la república en el contexto nacional.

Estos hechos proyectan circunstancias amenazantes a la espacialidad regional que había conformado desde años atrás la Universidad de Guadalajara, ya que la creación de universidades estatales parece tornarse en un factor de retención de la población procedente de otras entidades federativas. Sin embargo, enseguida veremos que los flujos migratorios prosiguen con proporciones similares.

\section{Crecimiento y diversificación de la matrícula universitaria}

En el lapso que corre entre 1960 y 1980 sobrevienen circunstancias que modifican el tamaño doméstico de la Universidad de Guadalajara. Tanto el crecimiento de la matrícula como la diversificación de la oferta educativa ayudan a dar paso a la configuración de una universidad más compleja y moderna. 
En esta etapa, la expansión educativa tiene comportamientos relativamente diferentes. Por ejemplo, en los años sesenta el crecimiento de la oferta educativa se debe más a la creación de nuevos espacios educativos que a la ampliación de la matrícula escolar. En ese decenio se crean las preparatorias 2, 3 y 4, y en el nivel superior se inauguran las carreras de agronomía y medicina veterinaria, y las carreras de ingeniero mecánico, ingeniero en electrónica, ingeniero electricista, ingeniero topógrafo e ingeniero industrial.

En los años setenta, por su parte, hay un fuerte crecimiento de la matrícula: de 36647 alumnos en 1970 se pasa a 192088 en 1980 (Oropeza, 1990); este engrosamiento de la población escolar se debe tanto a la apertura del ingreso como a la eclosión de nuevos establecimientos educativos. Así, en el medio superior se crean la Escuela Preparatoria para Trabajadores número 1, la Escuela Preparatoria número 5 y la Escuela Preparatoria para trabajadores número 2. En el nivel técnico se inauguran la Escuela de Enfermería de Ciudad Guzmán y la Escuela Politécnica de Ocotlán. En el nivel superior se abren las escuelas de turismo, psicología, geografía y diseño, la carrera de sociología y las facultades de administración, contaduría y trabajo social.

Este incremento permite el arribo de mayor número de estudiantes a los espacios universitarios, apertura que también se extiende para los jóvenes que provienen de otros estados del país.

\section{La espacialidad regional y la tenacidad de los flujos migratorios}

¿Qué revela la información en torno a la espacialidad regional de la Universidad de Guadalajara a lo largo de los años sesenta y setenta? La información muestra que la apertura de universidades estatales no retiene a los estudiantes en su localidad de origen, pero el crecimiento y diversificación de la oferta educativa de la Universidad de Guadalajara sí propicia algunos cambios en la elección profesional.

En la gráfica siguiente podemos ver que la procedencia de los alumnos prácticamente se mantiene igual a lo largo de los años sesenta y setenta: los estudiantes oriundos del estado de Jalisco pasan de $68 \%$ a $70 \%$, los que proceden de los estados del noroeste conservan la proporción de $12 \%$, los que vienen de los estados vecinos bajan de $9 \%$ a $8 \%$ y los que proceden de otras entidades y del extranjero pasan de $12 \%$ a $9 \%$.

Esta información muestra que más que un descenso relativo de la población proveniente de otras entidades, hay una estabilización de los flujos migratorios. Esta constancia de la población migrante permite la continuidad de la espacialidad regional que la Universidad de Guadalajara conforma desde mediados del siglo xx. Sin embargo, a la par de la continuidad de ciertos patrones de preferencias en esta población escolar, aparecen algunos cambios importantes en la distribución de la demanda educativa.

Con la apertura de nuevas opciones educativas se crea una mayor oferta que incide en la distribución de las afinidades de los estudiantes. En la gráfica siguiente podemos ver que al inicio de los años ochenta, dos de cada tres estudiantes procedentes de los estados vecinos se concentraban 
en las carreras de medicina (36\%), odontología (13\%), enfermería (9\%) y derecho (9\%). A pesar de esta concentración en estas cuatro carreras, observamos que el restante $33 \%$ se distribuye en un total de 16 carreras.

En la gráfica adjunta vemos que la ubicación de los estudiantes procedentes de los estados del noroeste no es muy diferente a la distribución anterior, ya que 51\% se ubica en las carreras de medicina y odontología, y $49 \%$ restante se distribuye en 21 carreras.

Todos estos datos revelan que las carreras vinculadas al campo de la salud atraen a la mayoría de los emigrantes de los estados vecinos y del noroeste, pero también muestran una creciente diversificación de las opciones elegidas. Esto es, a pesar de que la mayoría de los estudiantes de esas regiones optan principalmente por las carreras de medicina y odontología, un porcentaje importante tiende progresivamente a elegir otras carreras, generando cierta sincronía con la diversificación de la oferta educativa.

Así, apoyándonos en la espacialidad regional que se configura a partir de la procedencia de los estudiantes, se puede afirmar que la Universidad de Guadalajara sí erige un proyecto socioeducativo que abarca las regiones centro-occidente y noroeste del país. Es un fenómeno que se constituye tanto desde el polo de la oferta educativa como desde el polo que conforman los sujetos que demandan esos servicios; desde uno y otro se derivan influencias que inciden en la fisonomía que adquiere ese proyecto a lo largo del tiempo.

\section{Bibliografía}

Castañeda, Carmen (1994). "La función educativa de Guadalajara y su área de influencia en el occidente de México". Ricardo Ávila Palafox (coord.). El occidente de México en el tiempo. Guadalajara: Universidad de Guadalajara, , pp. 77-105.

Martínez Moctezuma, Lucía (2006). "Educando en las regiones: formación de profesores y prácticas educativas en el estado de Morelos, 1926-1934”. Lucía Martínez Moctezuma y Antonio Padilla Arroyo (coords.). Miradas a la historia regional de la educación. México: Miguel Ángel Porrua/Universidad Autónoma del Estado de Morelos, pp. 255-283.

Oropeza Sandoval, Luciano (1990). Estadística básica de la Universidad de Guadalajara, mimeo. Padilla Arroyo, Antonio (2006). "En torno a la construcción de las 'regiones educativas' en Morelos, 1871-1910". Lucía Martínez Moctezuma y Antonio Padilla Arroyo (coords.). Miradas a la historia regional de la educación. México: Miguel Ángel Porrua/Universidad Autónoma del Estado de Morelos, pp. 207-227. 
Piñera Ramírez, David (2008). "Las universidades de los estados de la república en el contexto nacional”. Leticia Pérez Puente y María de Lourdes Alvarado (coords.). Cátedras y catedráticos en la historia de las universidades e instituciones de educación superior en México, t. III. México: IISUE-UNAM.

Van Young, Eric (1989). La ciudad y el campo en el México del siglo XVIII. La economía rural de la región de Guadalajara, 1675-1820. México: Fondo de Cultura Económica.

Recibido: 02/01/15

Dictaminado: $12 / 06 / 15$

Corregido: $16 / 10 / 15$

Aceptado: 20/10/15 